\title{
An integrated approach for risk analysis based on CFD modelling and reliability engineering
}

\author{
V. C. Damaso ${ }^{1}$, P. A. M. Cabral ${ }^{1}$ \& L. A. Aguiar $^{2}$ \\ ${ }^{1}$ Brazilian Army Technological Centre (CTEx), Brazil \\ ${ }^{2}$ Centre for Environmental Systems Analysis - NASA (UFRJ/CNPq), \\ Brazil
}

\begin{abstract}
This work presents a methodology for the reduction of risks based on a reliability engineering study. In order to explain the methodology, a hypothetical case study is considered, where the scenario is the accidental release, into the environment, of a toxic chemical substance from a chemical process plant, leading to exposure of the local population. The dispersion is modelled by CFD-based software. The optimization process leads the calculated risk (individual and societal) to values below the tolerable limits in accordance with the supervisory boards. The connection between CFD, risk analysis and reliability calculations attests to the robustness and applicability of the proposed approach.
\end{abstract}

Keywords: risk analysis, CFD, reliability engineering, optimization.

\section{Introduction}

According to a comprehensive definition, risk can be defined as the product between the frequency of occurrence of a hazard scenario and the corresponding consequences, whenever this scenario may occur. Therefore, mitigation actions should reduce the frequency of undesirable events or their consequences. Industrial technicians are responsible for implementing and maintaining safe measures which, combined with maintenance rules, will provide lower failure probabilities for critical equipment/processes. Accidental and/or intentional releases of large amounts of hazardous chemicals into the atmosphere are actual possibilities, and emergency teams must be prepared to deal with these alarming situations. 
Risk analysis studies, concerning surrounding populations of industrial installations, have been considered another subject of great interest for environmental agencies, government, employers and scientific communities. Several researches have been conducted to put together computerized systems for risk analysis and emergency planning and some of these efforts can be cited as Alhajraf et al. [1]; Ermak et al. [2]; Lee et al. [3]; Mazzola [4] and Nasstrom et al. [5]. A system capable of predicting the dispersion of hazardous chemicals is a key tool to support emergency planning and an effective preparedness. Most of the risk assessment studies are conducted based on Gaussian plume model results, but some recent papers have shown the applicability of CFD (Computational Fluid Dynamics) models, although this tool is considered suitable only for preparedness and investigation in emergency response, due to its computational demand [6-10].

This work presents a methodology for the reduction of risks based on a reliability engineering study. In order to explain the methodology, a hypothetical case study is considered, where the scenario is the accidental release, into the environment, of a toxic chemical from a chemical process plant, leading to the exposure of the local population. A simplified version of this approach was presented by Damaso et al. [11], where only the individual risk was considered to establish a target for risk values. In this paper, both societal and individual risks are assessed and considered for defining the tolerable risk.

\section{Risk analysis}

Chemical process quantitative risk analysis (CPQRA) is a methodology designed to provide management with a tool to help evaluate overall process safety in the chemical process industry [12]. This analysis provides a quantitative method to evaluate risk and to identify areas for cost-effective risk reduction. CPQRA is a probabilistic methodology that is based on the NUREG procedures. Procedures for probabilistic risk analysis (PRA) have been defined in the PRA Procedures Guide [13] and the Probabilistic Safety Analysis Procedures Guide [14].

The basis of CPQRA is to identify incident scenarios and evaluate the risk by defining the probability of failure, the probability of various consequences and the potential impact of those consequences. The risk is defined in CPQRA as a function of probability or frequency and consequence of a particular accident scenario:

$$
\text { Risk }=F(s, c, f)
$$

where $s$ is hypothetical scenario; $c$ is estimated consequence(s); and $f$ is estimated frequency.

Considine [15] defines individual risk as the risk to a person in the vicinity of a hazard. This includes the nature of the injury to the individual, the likelihood of the injury occurring, and the time period over which the injury might occur. In this work, individual and societal risks are the criteria adopted to establish the tolerability limit. 
The calculation of individual risk at a geographical location near a plant assumes that the contributions of all incident outcome cases are additive. Thus, the total individual risk at each point is equal to the sum of individual risks, at that point, of all incident outcome cases associated with the plant, represented by eqn (2).

$$
I R_{x, y}=\sum_{i=1}^{n} I R_{x, y, i}
$$

where $I R_{x, y}$ is total individual risk of fatality at location $x, y\left(\mathrm{yr}^{-1}\right) ; I R_{x, y, i}$ is individual risk of fatality at location $x, y$ from incident outcome case $i\left(\mathrm{yr}^{-1}\right)$; and $n$ is the total number of incident outcome cases considered in the analysis.

The individual risk of fatality for each incident outcome case is obtained from:

$$
I R_{x, y, i}=F_{i} \cdot p_{f i}
$$

where $F_{i}$ is frequency of incident outcome case $i\left(\mathrm{yr}^{-1}\right)$, and $p_{f i}$ is the probability that incident outcome case $i$ will result in a fatality at location $x, y$.

In this work, the frequency of incident occurrence is based on the reliability of a safety system, which is directly associated to its availability along the mission time. The probability that the incident results in fatality is determined by the probit method $[12,16]$ and, in the case of a toxic release, the probit variable, $Y$, is of the form:

$$
Y=k_{1}+k_{2} \cdot \ln \left(\int_{t_{0}}^{t} C^{n} d t^{\prime}\right)
$$

where $k_{1}$ and $k_{2}$ are constants; $C$ is the concentration (ppm); $n$ is the concentration exponent; and $\left[t-t_{0}\right]$ is the exposure time $(\mathrm{min})$.

The probability of fatality, $p_{f}$, is given by:

$$
p_{f}=50\left[1+\frac{Y-5}{|Y-5|} \operatorname{erf}\left(\frac{|Y-5|}{\sqrt{2}}\right)\right]
$$

where $\operatorname{erf}$ is the error function.

Societal risk is a measure of risk to a group of people. The calculation of societal risk requires the same frequency and consequence information as individual risk. Additionally, societal risk estimation requires a definition of the population at risk around the facility. This definition can include the population type (e.g., residential, industrial, school), the likelihood of people being present, or mitigation factors [12].

A common form of societal risk is known as an F-N (frequency-number) curve. An F-N curve is a plot of cumulative frequency versus consequences (expressed as number of fatalities). A logarithmic plot is usually used because the frequency and number of fatalities range over several orders of magnitude.

The number of people affected by each incident outcome case is given by eqn (6):

$$
N_{i}=\sum_{x, y} P_{x, y} \cdot p_{f}
$$


where $N_{i}$ is the number of fatalities resulting from incident outcome case $i ; P_{x, y}$ is the number of people at location $x, y$; and $P_{f i}$ is as defined in eqn (3).

The number of people affected by all incident outcome cases must be determined, resulting in a list of all incident outcome cases, each with a frequency (from frequency analysis) and the number of people affected. This information must then be put in cumulative frequency form, according to eqn (7), in order to plot the F-N curve.

$$
F_{N}=\sum_{i} F_{i} \text { for all incident outcome case } i \text { for which } N_{i} \geq N
$$

where $F_{N}$ is the frequency of all incident outcome cases affecting $N$ or more people.

\section{Computational Fluid Dynamics (CFD)}

Several parameters need to be investigated in order to precisely understand the dispersion transport process of gases, droplets or particulates. These parameters are related to weather conditions such as wind speed and direction, cloud cover, relative humidity, temperature and stability class, the physical properties of the chemical itself such as density, volatility and phase, besides the location where the release may take place such as urban, rural or industrial areas. Classes of dispersion models are often defined according to the computational cost and degree of complexity [1]. Most of the risk assessment studies are conducted based on Gaussian plume models results, but some recent papers have shown the applicability of CFD (Computational Fluid Dynamics) models.

The CFD commercial package PHOENICS 2009, by CHAM, is used as a tool to obtain the corresponding concentration profiles. Numerical implementation of the boundary conditions is conducted by the use of the IN-FORM tool in PHOENICS 2009, which increases the simulation time, as higher computational effort is demanded. On the other hand, it represents a simpler and more intuitive manner of dealing with complex initial or boundary conditions. The same tool was also used to perform plume concentration tracking. Therefore, integration of concentration profiles could be calculated in each point of the discretization grid. A lighter-than-air gas is considered and a single density calculation is conducted for the whole domain, as a function of the gas fraction (mixture air and gas). A monitoring time of 10 minutes is simulated by the use of adequate time and space grids: 3000 time steps ( $0.2 \mathrm{~s}$ for each) and about 18,000 hexahedral cells.

\section{Availability modelling}

A preventive maintenance policy has an important role to achieve higher levels of availability in any industrial system. However, in many industrial plants, there are safety systems whose components are in standby mode. In standby mode, there is no way to know at first if the system's components are in operational condition or not. Hence, periodic tests should be scheduled so that hidden failures can be revealed and corrective maintenance actions be taken. 
Considering that a component has constant failure rate, the time-dependent reliability, $R(t)$, is given by:

$$
R(t)=e^{-\lambda t}
$$

The availability expression should consider the probability of the component being unavailable after the test and requiring repair. This probability, $P_{\tilde{A}}$, corresponds to the unavailability of the component just before initiating the test, added to the probability of failure of this component during the test, given that it was available in the beginning.

The component availability, $A(t)$, according to a policy of periodic tests [17], for a system in standby mode throughout the mission time, is given by:

$$
\begin{cases}A_{i}(t)=R_{i}(t)\left\{1-P_{\tilde{A}, i} \cdot \exp \left[-\mathrm{v} \cdot\left(t-\mathrm{T}_{i}\right)\right]\right\}, & \mathrm{T}_{i} \leq t<\mathrm{T}_{i+1}^{\prime} \\ A_{i}(t)=0, & \mathrm{~T}_{i+1}^{\prime} \leq t<\mathrm{T}_{i+1}\end{cases}
$$

where $i=1,2, \ldots, n ; n$ is the number of interventions; $v$ is the constant repair rate; and $\mathrm{T}^{\prime}{ }_{i+1} \leq \mathrm{t}<\mathrm{T}_{i+1}$ is the $i$-th test interval.

Identifying each $P_{\tilde{A}}$ with its corresponding cycle to the $i$-th test, one has

$$
P_{\tilde{A}, i}=1-A_{i-1}\left(t \rightarrow T_{i}^{\prime^{-}}\right) \frac{R_{i-1}\left(t \rightarrow T_{i}^{-}\right)}{R_{i-1}\left(t=T^{\prime}{ }_{i}\right)}
$$

where the notation $f\left(t \rightarrow x^{-}\right)$means the limit of the function $f(t)$ when $t$ tends to $x$ from the left. Thus, the mission availability of the component, $A^{*}$, is given by

$$
A^{*}\left(\mathrm{~T}_{\mathrm{f}}\right)=\frac{1}{\mathrm{~T}_{\mathrm{f}}} \int_{0}^{\mathrm{T}_{\mathrm{f}}} A(t) d t
$$

where $T_{\mathrm{f}}$ is the mission time.

\section{Case study}

The present case study deals with the risk assessment and management due to a possible toxic release from a chemical plant, which is expected to cause fatalities in a nearby population. In this study, the risk assessment cover a total area of $1,2 \mathrm{~km}^{2}(1000 \mathrm{~m} \times 1200 \mathrm{~m})$, and the release point has the coordinates $(\mathrm{x}=350 \mathrm{~m}, \mathrm{y}=560 \mathrm{~m})$ as shown in fig. 1 . The accidental scenario considered in this study is the catastrophic rupture of a tank containing a gaseous toxic industrial chemical (TIC), caused by the failure of the pressure control system of the tank. After the rupture, a toxic cloud is formed instantly and dragged by the wind over the local population. A simplified drawing of the pressure control system of the tank is also shown in fig. 1. The rupture will happen if an overpressure occurs in the tank and, simultaneously, the pressure control system fails.

The region is subjected to distinct climatic conditions with respective wind regimes along the year. The wind regime that has significant influence on the risk to the local population can be simplified (table 1), considering representative average values. 
The CFD simulation is conducted solely considering obstacles such as some equipment, buildings and devices present in the surroundings of the source point. No complex terrain is taken under consideration for the gas flow. Most puff models consider stability classes as there are changes for the dispersion coefficients. Here, different turbulence models, with different eddy diffusivities, are used to represent conditions of low turbulence level, leading to a more persistent condition at night, and the opposite during a day release. The two periods (day and night) subjected to their respective wind regimes produce six possible scenarios, as shown in table 1.

The pressure control system is considered failed for causing the catastrophic rupture of the tank if the main feeding line to the neutralizer is unavailable. In other words, the failure occurs if at least one of the following components fails: PIC (pressure indicator controller) and valves 1 to 4 . Thus, the system's

Table 1: Percentage of the interest wind regimes along the year and postulated scenarios of dispersion.

\begin{tabular}{ccccc}
\hline Scenario & Velocity $(\mathrm{m} / \mathrm{s})$ & Direction (going to) & $\%$ (yearly) & Period \\
\hline 1 & 3.0 & S - N & 6.0 & day \\
2 & 3.0 & SE - NW & 8.0 & day \\
3 & 2.5 & E - W & 5.0 & day \\
4 & 2.0 & S - N & 9.5 & night \\
5 & 2.0 & SE - NW & 7.5 & night \\
6 & 1.5 & E - W & 4.2 & night \\
\hline
\end{tabular}

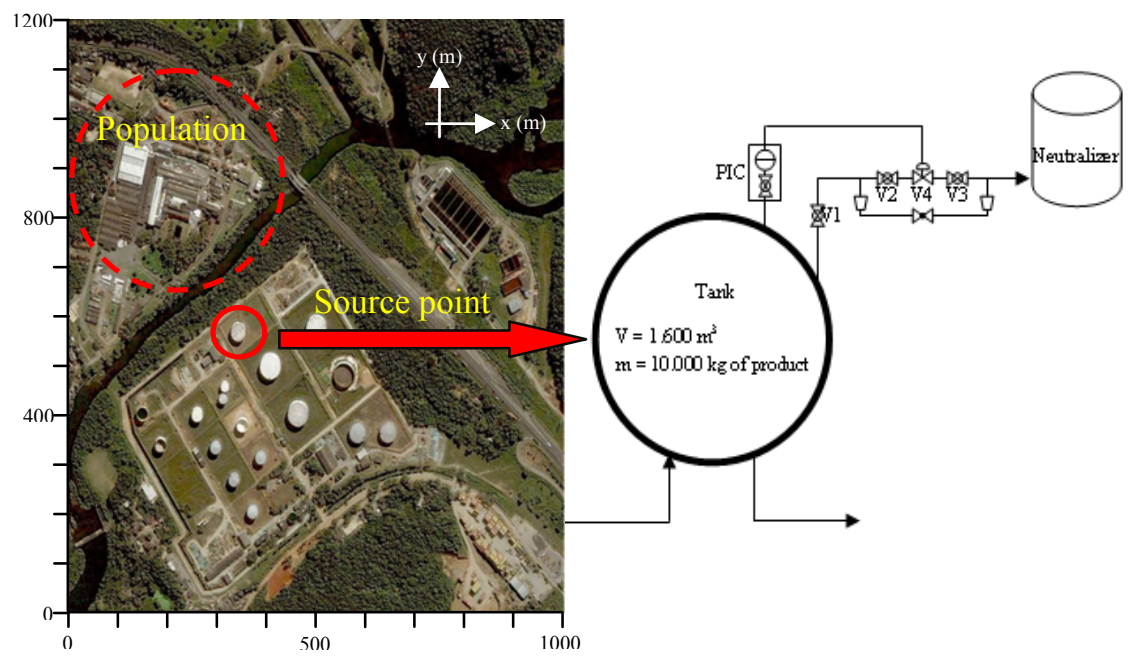

Figure 1: Case study scenario, where North points to the top of the image with schematic view of the tank and safety system. 
availability, $A_{\text {sys }}(t)$, corresponds to the product of the availabilities of all components:

$$
A_{s y s}(t)=\prod_{j=1}^{m} A_{j}(t)
$$

where $m$ is the number of components. The failure and repair rates for the components are present in table 2 .

Table 2: $\quad$ Parameters of the system's components.

\begin{tabular}{ccc}
\hline Component & $\lambda\left(\right.$ day $\left.^{-1}\right)$ & $v\left(\right.$ day $\left.^{-1}\right)$ \\
\hline PIC & $1.00 \times 10^{-5}$ & 0.85 \\
V 1 & $4.56 \times 10^{-5}$ & 1.70 \\
V 2 & $4.30 \times 10^{-5}$ & 1.50 \\
V 3 & $4.30 \times 10^{-5}$ & 1.50 \\
V 4 & $2.14 \times 10^{-6}$ & 1.05 \\
\hline
\end{tabular}

For scenario $i$, the risk is computed as

$$
I R_{x, y, i}=F_{o p} \cdot\left(1-A_{s y s}^{*}\left(T_{f}\right)\right) \cdot p_{f i}
$$

The frequency of overpressure occurrence, $F_{o p}$, is estimated in $3.5 \times 10^{-4}$ per year. For the TIC, the probit parameters are: $k_{1}=-19.27 ; k_{2}=3.69 ; n=1$. The availability calculations are done over a period of one year $\left(T_{f}=1 \mathrm{yr}\right)$ and the test downtime for all components is one day. It is considered that the Regulatory Board imposes the limit of $1 \times 10^{-4} \mathrm{yr}^{-1}$ for the tolerable and $1 \times 10^{-6} \mathrm{yr}^{-1}$ for the negligible total risk. It is important to note that, for the total risk calculation, the value obtained by eqn (13) must be multiplied by the yearly fraction of the wind and by 0.5 (referring to day or night period).

The coordinates of the populated area considered in the societal risk calculation and the respective population density for each scenario is presented in table 3.

Table 3: The coordinates of the populated area and the respective population density for each scenario.

\begin{tabular}{cllc}
\hline Area & \multicolumn{1}{c}{$\begin{array}{c}\text { Coordinates } \\
(\mathrm{m})\end{array}$} & \multicolumn{2}{c}{$\begin{array}{c}\text { Population density } \\
\left(\text { persons } / \mathrm{m}^{2} \text { ) }\right.\end{array}$} \\
& \multicolumn{1}{c}{ day } & night \\
\hline A & $\begin{array}{l}\mathrm{x}=0 \text { to } 157.89 \\
\mathrm{y}=1002.53 \text { to } 1154.43\end{array}$ & 0.002293 & 0.003127 \\
& $\mathrm{x}=52.63$ to 210.53 & 0.004169 & 0.002085 \\
B & $\begin{array}{l}\mathrm{y}=744.30 \text { to } 987.34 \\
\mathrm{x}=236.84 \text { to } 421.05\end{array}$ & 0.002010 & 0.001340 \\
& $\mathrm{y}=744.30$ to 987.34 & & 0.003475 \\
\hline
\end{tabular}




\section{Results}

In order to reduce the total risk, an optimization study was performed aiming to establish a test policy which minimizes the system`s unavailability. According to the best solution found by the optimization process, tests should be carried out obeying intervals of 120 days.

For comparison purposes, individual and societal risks were calculated using values to the system's mission unavailability in two different conditions: without tests, $\tilde{A}^{*}\left(\mathrm{~T}_{\mathrm{f}}\right)=0.0258$, and with 120 day intervals between tests, $\tilde{A}^{*}\left(\mathrm{~T}_{\mathrm{f}}\right)=0.0142$.

Table 4 presents the results for the coordinates in the populated area with highest individual risk according to both without test and optimized test scheduling conditions.

Table 4: $\quad$ Results for without test and optimized test scheduling conditions.

\begin{tabular}{ccc}
\hline Condition & $\begin{array}{c}\text { System's } \\
\text { unavailability }\end{array}$ & $\begin{array}{c}\text { Highest individual risk } \\
\text { in the populated area }\end{array}$ \\
\hline no test & 0.0258 & $1.3238 \times 10^{-06}$ \\
120 day intervals & 0.0142 & $7.2862 \times 10^{-07}$ \\
\hline
\end{tabular}

A spreadsheet generated using Microsoft Excel software was used for viewing the individual risk, applying the same discretization used in the CFD modelling to the studied area. Figure 2 shows the distribution of risk associated with accidental scenario.


Figure 2: Distribution of risk associated with accidental scenario with optimized test scheduling and with test. 
The F-N curves are showed in fig.3. In the condition without test, some points of the plotted curve are in the intolerable risk region, while in the optimized condition, this problem is solved.

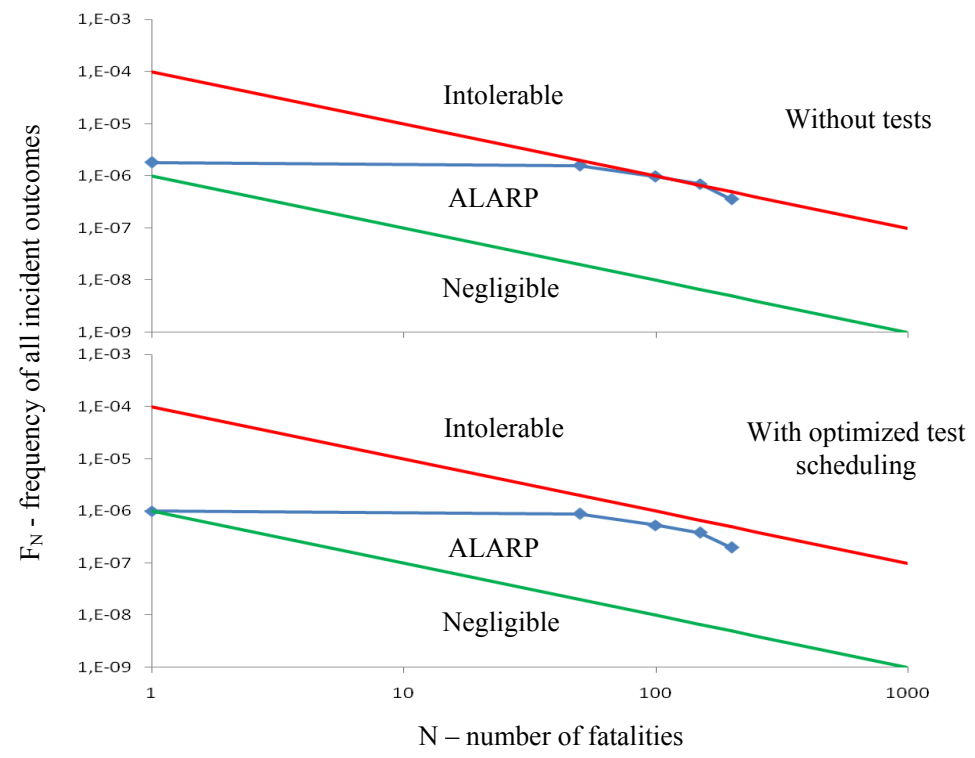

Figure 3: F-N curves to conditions without test and with optimized test scheduling.

\section{Conclusion}

CFD calculations tend to be more realistic than other models commonly used to simulate gas dispersion. Due to the extra computational cost demanded by the use of this kind of model, in contrast with puff models, it would be recommended only for preparation and investigation, not for online monitoring (unless a very fast computation system is available).

In terms of reliability engineering, the results are fully satisfactory and compatible with the system studied, demonstrating that the presented approach, based on improvement of safety systems availability, has effectiveness in order to reduce the inherent risk.

As extension of the accomplished work, several other factors should be considered, like aging, redundancy allocation and costs, besides expanding the studied system in a greater number of components. In this case, non-constant failure rates should be considered and optimization tools can be used for performance optimization. For dealing with aging systems, the study should cover more extensive periods (e.g. 10 years) in order to allow for a more accurate 
observation of aging effects. Further work is underway to consider larger number of scenarios and other effects, like overpressure and thermal radiation.

The connection between CFD, risk assessment and reliability calculations attest to the robustness and the applicability of the proposed methodology.

\section{Acknowledgement}

The authors are very grateful to CTEx (Brazilian Army Technological Centre) and the Centre for Environmental Systems Analysis for supporting this work.

\section{References}

[1] Alhajraf, S., Al-Awadhi, L., Al-Fadala, S., Al-Khubaizi, A., Khan, A. R. \& Baby, S., Real-time response system for the prediction of the atmospheric transport of hazardous materials, Journal of Loss Prevention in the Process Industries, vol 18, 520-525, 2005.

[2] Ermak, D. L., Sugiyama, G. \& Nasstrom, J. S., Atmospheric release assessment program (ARAP) science and technology base development, Lawrence Livermore National Laboratory. UCRL-JC-148450, 2002.

[3] Lee, R. L., Albritton, J. R., Ermak, D. L. \& Kim, J., Computational fluid dynamics modeling for emergency preparedness and response, Regional atmospheric science division, Lawrence Livermore National Laboratory. UCRL-JC-120469, 1995.

[4] Mazzola, C., Atmospheric dispersion modeling resources, 2nd Ed., United States Department of Energy, 1995.

[5] Nasstrom, J. S., Sugiyama, G., Leone Jr., J. M. \& Ermak, D. L., A real-time atmospheric dispersion modeling system, Proc. of the 11th Joint Conference on the application of air pollution meteorology with the air and waste management association. January 9-14, Long Beach, CA., 2000.

[6] Hjertager, B. H. \& Solberg, T., A review of computational fluid dynamics (CFD) modeling of gas explosions, Prevention of Hazardous Fires and Explosions, 77-91, Kluwer Academic Publishers, 1999.

[7] Mukai, S., Suzuki, J., Mitsuishi, H., Oyakawa, K. \& Watanabe, S., CFD Simulation on diffusion of leaked hydrogen caused by vehicle accident in tunnels, Proc. of the First International Conference on Hydrogen Safety, 810 set, Pisa, Italy, 2005.

[8] Gallego, E., Garcia, J., Migoya, E., Crespo, A., Kotchourko, A., Yanez, J. \& Beccantini, A., An inter-comparison exercise on the capabilities of CFD models to predict deflagration of a large-scale H2-Air mixture in open atmosphere, Proc. of the First International Conference on Hydrogen Safety, 8-10 set, Pisa, Italy, 2005.

[9] Wilkening, H. \& Baraldi, D., CFD Modelling of accidental hydrogen release from pipelines, International Journal of Hydrogen Energy, vol 32, 2206-2215, 2007.

[10] Venetsanos, A. G., Baraldi, D., Adams, P., Heggem, P. S. \& Wilkening, H., CFD Modelling of hydrogen release, dispersion and combustion for 
automotive scenarios, Journal of Loss Prevention in the Process Industries, vol 21, 162-184, 2008.

[11] Damaso, V.C., Okamoto, R.M. \& Cabral, P.A.M., An integrated approach for risk management based on systems availability, Reliability, Risk and Safety - Theory and Applications, Proc. of the European Safety and Reliability Conference (ESREL 2009), Vol. 2, 953-958, Prague, Czech Republic, Sept 7-10, 2009.

[12] AIChE/CCPS, Guidelines for Chemical Process Quantitative Risk Analysis. Center for Chemical Process Safety, American Institute of Chemical Engineers, 2nd ed., New York (ISBN 0-8169-0720-X), 2000.

[13] NUREG, PRA Procedures Guide: A Guide to the Performance of Probabilistic Risk Assessment for Nuclear Power Plants. 2 volumes, NUREG/CR-2300, U.S. Nuclear Regulatory Commission, Washington, D.C. January, 1983 (available from NTIS). 1983.

[14] NUREG, Probabilistic Safety Analysis Procedures Guide, NUREG/CR2815. Nuclear Regulatory Commission, Washington D.C. August, 1985 (available from NTIS), 1995.

[15] Considine, M., The Assessment of Individual and Societal Risks. SRD Report R-310, Safety and Reliability Directorate. Warrington: UK Atomic Energy Authority, 1984.

[16] Finney, D. J. (1971). Probit Analysis. 3rd ed. London: Cambridge University Press. (ISBN 0-521-080-41-X).

[17] Damaso, V. C., An integrated optimization modeling for safety system availability using genetic algorithms, D.Sc. Thesis, Federal University of Rio de Janeiro, Nuclear Engineering Program, 2006 [in Portuguese]. 